\title{
Volumetria de Genipa americana em plantio homogêneo no Sudoeste da Bahia
}

\author{
Mislene Barbosa Rocha ${ }^{1}$, Patrícia Anjos Bittencourt Barreto-Garcia ${ }^{1 \star}$, Wilmerson Bernardino Prado ${ }^{1}$, Alessandro de Paula ${ }^{1}$, \\ Valdemiro Conceição Júnior ${ }^{1}$
}

${ }^{1}$ Universidade Estadual do Sudoeste da Bahia, Estrada do Bem Querer, Km 4, CEP 45083-900, Vitória da Conquista, BA, Brasil

*Autor correspondente:

patriciabarreto@uesb.edu.br

Termos para indexação:

Jenipapo

Modelos volumétricos

Fator de forma

Index terms:

Jenipapo

Volumetric models

Form fator

\begin{abstract}
Resumo - O objetivo deste trabalho foi avaliar estimativas volumétricas para a espécie Genipa americana L., conhecida vulgarmente como jenipapeiro, em plantio puro no município Vitória da Conquista, BA. Foram cubadas rigorosamente 100 árvores em pé para determinação do volume individual. De posse dos dados da cubagem foram ajustados dez modelos volumétricos. Os melhores modelos foram selecionados com base no critério de seleção do valor ponderado dos escores dos parâmetros estatísticos e distribuição de resíduos. As estimativas de volume foram realizadas por fator de forma e por equações ajustadas e os resultados foram comparados ao volume obtido com a cubagem, para validação das estimativas. Dentre os métodos utilizados para predição do volume, o mais recomendado é o emprego de equações volumétricas ajustadas. O modelo que apresentou melhor desempenho para estimar o volume total foi o de Spurr (Log).
\end{abstract}

\section{Volumetry of Genipa americana in homogeneous plantation in Southwest Bahia}

\begin{abstract}
This research aim to evaluate the volumetric estimates obtaining for Genipa americana, commonly known as jenipapeiro, in pure plantation in the municipality of Vitória da Conquista, Bahia State, Brazil. To determine individual volume, 100 standing trees were rigorously cubed. Ten volumetric models were adjusted. The best models were selected based on the selection criteria of weighted value of statistical parameters scores and residues distribution. Volume estimates were obtained by form factor and by adjusted equations. To validate the estimates, the calculate volumes were compared to measured data. Among the used methods to predict wood volume, the adjusted volumetric equations are recommended. Spurr (Log) model present the best performance to estimate total wood volume.
\end{abstract}

\section{Introdução}

Nativa do Brasil, a espécie Genipa americana L. pertence à família Rubiaceae, sendo conhecida vulgarmente em todo o país como jenipapeiro. Quando adulta, é uma árvore de fuste reto e alto, com dominância apical bem definida. Possui importância econômica, tanto pela utilização dos frutos, quanto por sua essência florestal, com grande potencial na recomposição de matas ciliares e para a produção de madeira para confecção de diversos produtos, como peças curvadas (cabos de ferramentas, obras de torno, batentes de portas e janelas, etc.), carpintaria em geral, construção civil e naval (Lorenzi, 2002).

Apesar da comprovada importância do jenipapeiro, o conhecimento a seu respeito ainda é incipiente, principalmente no que se refere aos seus aspectos silviculturais e dendrométricos, o que evidencia a 
relevância de estudos relacionados ao tema. Segundo Tonini et al. (2005), em geral, grande parte dos trabalhos que envolvem o estudo de variáveis dendrométricas são relacionados a espécies exóticas, de rápido crescimento, a exemplo dos gêneros Pinus e Eucalyptus, por estas despertarem maior interesse que as espécies nativas, em decorrência da maior disponibilidade de informações acerca da sua biometria, ecologia e silvicultura. No entanto, a compreensão do potencial produtivo e estoque madeireiro desses povoamentos tornam-se fundamental para o correto manejo, planejamento e sustentabilidade da produção.

A estimativa da produção volumétrica de madeira de um povoamento florestal normalmente é realizada por meio de inventário florestal, utilizando-se uma amostra como base para se fazer inferências sobre seus parâmetros, tais como diâmetro, altura, volume e número de árvores por hectare (Machado et al., 2000). No que se refere ao volume, a cubagem rigorosa e, posteriormente, o emprego dos seus dados para o ajuste de equações de regressão ou para obtenção do fator de forma, constituem as técnicas mais comumente utilizadas (Soares et al., 2011). De acordo com Guimarães \& Leite (1996), o emprego de equações volumétricas é o procedimento mais eficiente. No entanto, ainda que muitos modelos sejam eficientes, nem sempre os mesmos se ajustam a todas as espécies e condições, fazendo-se necessário testá-los por meio de estatísticas apropriadas, identificando o melhor para cada situação.

O presente trabalho teve como objetivo avaliar a obtenção de estimativas volumétricas para a espécie Genipa americana em plantio homogêneo.

\section{Material e métodos}

\section{Descrição da área}

O estudo foi conduzido em um povoamento homogêneo de Genipa americana, com seis anos de idade, localizado no Área Experimental da Universidade Estadual do Sudoeste da Bahia (UESB), campus de Vitória da Conquista, BA, cujas coordenadas geográficas são: $-14^{\circ} 53^{\prime} 0,89^{\prime \prime} \mathrm{S}$ e $-40^{\circ} 47^{\prime} 50,58^{\prime \prime} \mathrm{W}$.

A região possui relevo plano a levemente ondulado, com altitude média de $840 \mathrm{~m}$. O clima é tropical de altitude (Cwb), segundo a classificação de Köppen, com valores médios de temperatura e precipitação de $25^{\circ} \mathrm{C}$ e $850 \mathrm{~mm}$, respectivamente (Oliveira et al., 2013). O solo da área estudada é classificado como Latossolo Amarelo Distrófico.

\section{Coleta de dados}

Foram selecionadas 100 árvores, distribuídas em quatro classes diamétricas, com amplitude correspondendo ao desvio padrão dos valores obtidos de diâmetro a 1,30 m do nível do solo $(2,5 \mathrm{~cm})$ (Tabela 1), conforme proposto por Scolforo \& Figueiredo Filho (1998).

Tabela 1. Distribuição diamétrica das árvores do povoamento de Genipa americana.

\begin{tabular}{cccc}
\hline $\begin{array}{c}\text { Classe de } \\
\text { diâmetro }\end{array}$ & Centro de classe & Frequência & $\begin{array}{c}\mathbf{N}^{\circ} \text { de } \\
\text { árvores } \\
\text { selecionadas }\end{array}$ \\
\hline $3,20-5,69$ & 4,4 & 9 & 0 \\
$5,70-8,19$ & 6,9 & 66 & 30 \\
$8,20-10,69$ & 9,4 & 87 & 31 \\
$10,70-13,19$ & 11,9 & 39 & 25 \\
$13,20-15,70$ & 14,5 & 14 & 14 \\
\hline Total & & 215 & 100 \\
\hline
\end{tabular}

A cubagem foi realizada em árvores em pé, com auxílio de escada, por meio do método de Smalian. Em cada árvore, além da altura total, foram medidas as circunferências com casca nas posições $0,1 \mathrm{~m}, 0,8 \mathrm{~m}, 1,3$ $\mathrm{m}, 2,1 \mathrm{~m}$ e a partir desse ponto de metro em metro até o diâmetro limite de 5,0 cm com casca. Após a medição da última seção de um metro, obteve-se também o comprimento da ponta.

As medidas de circunferências e alturas das árvores cubadas foram obtidas com auxílio de fita métrica, trena e hipsômetro eletrônico. Após a transformação das circunferências para diâmetros, foram calculados os volumes por seção. $\mathrm{O}$ volume total foi obtido pelo somatório dos volumes de todas as seções mais o volume da ponta (calculado com base na fórmula do cone).

\section{Modelos volumétricos}

Foram testados dez modelos selecionados na literatura, sendo oito lineares e dois não lineares (Tabela 2), que apresentam como variável dependente o volume (V) em função das variáveis independentes, diâmetro a 1,3 m do nível do solo (DAP) e altura total $(\mathrm{H})$. 
Tabela 2. Modelos volumétricos testados.

\begin{tabular}{ccc}
\hline $\mathbf{N}^{\circ}$ & \multicolumn{1}{c}{ Modelo } & Autor* \\
\hline 1 & $\operatorname{Ln}(\mathrm{V})=\beta 0+\beta 1 * \operatorname{Ln}(\mathrm{DAP})+\varepsilon$ & Hush \\
2 & $\mathrm{~V}=\beta 0+\beta 1 * \mathrm{DAP}^{2}+\varepsilon$ & Kopezky-Gerhardt \\
3 & $\mathrm{Ln}(\mathrm{V})=\beta 0+\beta 1 * \operatorname{Ln}(\mathrm{H})+\beta 2 * \operatorname{Ln}(\mathrm{DAP})+\varepsilon$ & $\begin{array}{c}\text { Schumacher-Hall } \\
(\text { Log })\end{array}$ \\
4 & $\mathrm{~V}=\beta 0+\beta 1 * \mathrm{DAP} * \mathrm{H}+\varepsilon$ & Spurr linear \\
5 & $\mathrm{~V}=\beta 0+\beta 1 * \mathrm{DAP}+\varepsilon$ & Linha reta \\
6 & $\mathrm{~V}=\beta 0+\beta 1 * \mathrm{DAP}+\beta 2 * \mathrm{DAP}^{2}+\varepsilon$ & Hohenald-Krenm \\
7 & $\mathrm{Ln}(\mathrm{V})=\beta 0+\beta 1 * \operatorname{Ln}\left(\mathrm{DAP} \mathrm{P}^{2} \mathrm{H}\right)+\varepsilon$ & Spurr $(\log )$ \\
8 & $\mathrm{Ln}(\mathrm{V})=\beta 0+\beta 1 * \operatorname{Ln}(\mathrm{DAP})+\beta 2 * 1 / \mathrm{DAP}+\varepsilon$ & Brenac \\
9 & $\mathrm{~V}=\beta 0 * \mathrm{DAP}^{\beta 1} * \mathrm{H}^{\beta 2}+\varepsilon$ & Schumacher-Hall \\
10 & $\mathrm{~V}=\beta 0 * \mathrm{DAP}^{2} * \mathrm{H}^{\beta 1}+\varepsilon$ & Spurr \\
\hline
\end{tabular}

Em que: $\beta_{\mathrm{i}}=$ coeficiente dos modelos; DAP = diâmetro com casca a 1,30 $\mathrm{m}$ do nível do solo (cm); $\mathrm{H}=$ altura total (m); $\mathrm{Ln}=$ Logaritmo neperiano. *Modelos citados nas seguintes fontes: Finger (1992), Azevedo et al. (2011) e Soares et al. (2011).

Para a seleção dos modelos, os critérios estatísticos considerados foram: coeficiente de determinação ajustado $\left(\mathrm{R}^{2}{ }_{\mathrm{aj}}\right)$; erro padrão da estimativa em percentagem $\left(\mathrm{S}_{\mathrm{yx}} \%\right)$; critério de informação de Akaike (AIC); critério de informação Bayesiano (BIC) e valor ponderado dos escores dos parâmetros estatísticos (VP). Os modelos foram ajustados empregando-se o programa Statgraphics ${ }^{\circledR}$ Centurion XVI e planilha eletrônica.

Nos modelos em que a variável dependente sofreu algum tipo de transformação logarítmica, o erro padrão foi recalculado na escala original, para possibilitar a comparação com os modelos aritméticos (Machado, 2002). Assim, previamente, multiplicou-se o volume estimado pelo fator de correção de Meyer, conforme fórmulas (1) e (2)

Fator de Meyer $=\mathrm{e}^{0,5 * \mathrm{Syx}}$

Onde: $\mathrm{e}=2,718281828$; Syx $=$ erro padrão da estimativa $\left(\mathrm{m}^{3}\right)$.

$$
\text { Syx recalc. }=\sqrt{\frac{\sum \text { SQres. }}{n-p}}
$$

Onde: Syx recalc = Erro padrão recalculado; SQres. = soma do quadrado do resíduo; $\mathrm{n}=$ número de dados; $\mathrm{p}=$ número de variáveis independentes do modelo; $\bar{X}$ = média do volume.

O AIC admite a existência de um modelo "real" que descreve os dados e que é desconhecido, oferecendo uma medida relativa das informações perdidas, quando um determinado modelo é usado para descrever a realidade. O BIC descreve a relação entre a variável dependente e as diversas variáveis explanatórias, maximizando a probabilidade de se identificar o verdadeiro modelo dentre os avaliados.

Os valores de AIC e BIC foram calculados a partir das expressões (3), (4) e (5). Quando a soma dos quadrados dos erros aumenta, o AIC e o BIC também aumentam, mas ambos os critérios penalizam a adição de parâmetros, buscando a seleção de um modelo que se ajusta bem com um mínimo de parâmetros (simplicidade e parcimônia). Assim, quanto menor o valor de AIC e BIC, melhor o ajuste (Carvalho et al., 2011; Miguel et al, 2014).

$$
\begin{aligned}
& \mathrm{AIC}=-2 \mathrm{Ln}(\mathrm{L})+2 \mathrm{k} \\
& \mathrm{BIC}=-2 \mathrm{Ln}(\mathrm{L})+\mathrm{Ln}(\mathrm{n}) \mathrm{k} \\
& L=\frac{\sum e^{2}}{n}
\end{aligned}
$$

Onde: $\mathrm{AIC}=$ critério de informação de Akaike; $\mathrm{BIC}=$ critério de informação Baeysiano; $\mathrm{L}=$ máxima verossimilhança; $\mathrm{e}=$ erro; $\mathrm{k}=$ número de parâmetros do modelo; $\mathrm{n}=$ número de observações.

$\mathrm{O}$ valor ponderado dos escores dos parâmetros estatísticos (VP) leva em consideração os critérios citados anteriormente, como uma síntese dos resultados a partir da atribuição de pesos às variáveis estatísticas, que foram ordenadas de acordo com o seu nível de precisão. Assim, foi atribuído peso 1 para a melhor equação, 2 para a segunda melhor e assim por diante (ranking), conforme a metodologia proposta por Thiersch (1977) e adotada por Azevedo et al. (2011). Após a classificação individual, foi somada a pontuação de cada modelo matemático, sendo selecionados os dois melhores modelos.

Os modelos selecionados com base no VP foram analisados quanto à distribuição gráfica dos resíduos, com propósito de verificar a ocorrência de tendências nas estimativas, e quanto à amplitude relativa dos resíduos (AR), obtida pela diferença entre o maior e o menor resíduo gerado pela equação.

\section{Fator de forma}

Além dos modelos volumétricos, também foi avaliado o emprego do fator de forma $(f)$, razão entre o volume real e o volume do cilindro com o DAP, como método para estimativa do volume total de madeira da árvore. Assim, os volumes individuais foram obtidos com base na média aritmética dos valores individuais de $f$, a qual foi multiplicada pelo volume do cilindro. 


\section{Validação dos resultados}

Com objetivo de avaliar como as equações ajustadas e o fator de forma comportam-se na estimativa de dados independentes daqueles usados no ajuste, foi realizada a validação dos dados. Para isso, foram retiradas $10 \%$ das árvores (10 árvores) que compunham o conjunto total de dados. A seleção das árvores foi feita de forma aleatória.

A validação consistiu em estimar os volumes das 10 árvores inicialmente separadas do grupo total de árvores disponíveis para o ajuste e, posteriormente, em comparar estas estimativas com os volumes reais das árvores (volume da cubagem). Para isso, foi aplicado o teste $\mathrm{t}$ para dados pareados $(\alpha=0,01)$, utilizandose o programa estatístico $S A E G \AA$ v.9.1. Esse método é tradicionalmente utilizado para examinar se uma técnica de predição é igual ou pode substituir outra já existente, conforme adotado por Rezende et al. (2006). De forma complementar, foi considerado o erro médio em percentagem, calculado pela diferença entre valor real (medido) e o valor estimado por ambas as equações ajustadas e pelo fator de forma, como empregado por Kohler et al. (2013).

\section{Resultados e discussão}

Os modelos volumétricos apresentaram coeficiente de determinação ajustado $\left(\mathrm{R}_{\mathrm{aj}}^{2}\right)$ variando de 86,0 a $95,6 \%$. Apenas para um dos modelos testados o $\mathrm{R}^{2}{ }_{\text {aj }}$ foi inferior a $88 \%$, o que demonstra, de modo geral, um bom desempenho estatístico dos modelos testados pelo elevado grau de ajuste da variável dependente em função das variáveis independentes. O maior valor para essa estatística foi verificado no modelo 3 (Schumacher-Hall (Log)) (Tabela 3).

Rocha et al. (2010), Azevedo et al. (2011) e Martins et al. (2015), testando equações volumétricas para diferentes espécies de Eucalyptus, verificaram maiores valores de $\mathrm{R}_{\text {aj }}^{2}$ para a equação de Schumacher-Hall (Log). Martins et al. (2015) atribuíram esse resultado à característica de dupla entrada do modelo, que permite a obtenção de estimativas a partir de duas variáveis independentes (DAP e altura total) e, consequentemente, uma melhor representação da variação real da população amostrada.

Tabela 3. Coeficientes e parâmetros estatísticos obtidos no ajuste dos modelos para o volume total de Genipa americana.

\begin{tabular}{|c|c|c|c|c|c|c|c|c|c|}
\hline Modelos & $\beta 0$ & $\beta 1$ & $\beta 2$ & $\beta 3$ & $\mathbf{R}_{\mathrm{aj}}^{2}(\%)$ & $S_{y x}(\%)$ & AIC & BIC & VP \\
\hline 1 & $-8,94181$ & 2,12010 & & & 93,29 & 21,49 & $-8,07$ & $-3,07$ & 15 \\
\hline 2 & $-0,00143$ & 0,00019 & & & 88,34 & 21,36 & $-7,44$ & $-2,44$ & 22 \\
\hline 3 & $-8,79235$ & 0,52241 & 1,65285 & & 95,57 & 17,71 & $-4,99$ & 2,51 & 22 \\
\hline 4 & 0,00530 & 0,00002 & & & 91,36 & 18,38 & $-7,73$ & $-2,73$ & 18 \\
\hline 5 & $-0,02135$ & 0,00405 & & & 86,01 & 23,39 & $-9,35$ & $-4,35$ & 22 \\
\hline 6 & 0,00026 & $-0,00033$ & 0,00021 & & 88,22 & 21,47 & $-5,37$ & 2,13 & 32 \\
\hline 7 & $-8,67124$ & 0,72279 & & & 95,26 & 17,73 & $-7,14$ & $-2,14$ & 16 \\
\hline 8 & $-10,27530$ & 2,52903 & 3,76916 & & 93,31 & 21,65 & $-6,07$ & 1,43 & 26 \\
\hline 9 & 0,00015 & 1,60397 & 0,59838 & & 92,10 & 17,58 & $-4,25$ & 3,25 & 26 \\
\hline 10 & 0,00016 & 0,73004 & & & 92,01 & 17,68 & $-6,38$ & $-1,38$ & 21 \\
\hline
\end{tabular}

Em que: $\beta \mathrm{i}=$ coeficientes dos modelos; $\mathrm{R}_{\mathrm{aj}}^{2}=$ coeficiente de determinação ajustado; $\mathrm{S}_{\mathrm{yx}}=$ erro-padrão da estimativa; $\mathrm{n}=90$.

O erro padrão da estimativa $\left(\mathrm{S}_{\mathrm{yx}} \%\right)$ apresentou valores entre 17,6 e $23,4 \%$. Os menores resultados para esta estatística foram encontrados nos modelos 9 e 10 (Tabela 3), os únicos não lineares testados (Schumacher-Hall e Spurr, respectivamente), o que indica que estas funções propiciam, em média, menor distância entre os valores estimados e os observados. De acordo com Rangel et al. (2006), previsões da produção de madeira a partir de estimativas de uma função não linear tendem a ser melhores, por apresentarem menores erros padrões residuais.

Em geral, não se verificou uma relação nítida entre os valores de $\mathrm{R}_{\text {aj }}^{2}$ e os valores de $\mathrm{S}_{\mathrm{yx}} \%$ dos modelos avaliados, embora seja comum verificar correlação entre estes parâmetros, onde os tratamentos com piores valores para um são também os de menor desempenho 
para o outro (Barros et al., 2002). De forma semelhante ao observado no presente trabalho, Scolforo et al. (2004) observaram valores altos de Syx \% e, ao mesmo tempo, valores satisfatórios de $\mathrm{R}^{2}$ aj. Os autores relacionaram esse resultado com a variabilidade encontrada nas árvores-amostra.

De modo geral, com base nos valores de $\mathrm{R}_{\text {aj }}^{2}$ e de $\mathrm{S}_{\mathrm{yx}}$, verificou-se bom ajuste tanto de modelos que utilizaram apenas DAP como variável independente quanto daqueles que empregaram DAP e altura. De acordo com Higuchi et al. (1998), os modelos de equações alométricas com apenas uma variável independente (DAP) podem apresentar resultados tão consistentes quanto os modelos que também utilizam a altura $(\mathrm{H})$.

Em relação aos critérios AIC e BIC, os melhores resultados foram observados nos modelos 1 (Hush) e 5 (linha reta), que utilizam apenas o DAP como variável independente.

De acordo com o valor ponderado (VP) (Tabela 3), os modelos de melhor desempenho foram: 1 (Hush) e 7 (Spurr (Log)). Ribeiro et al. (2014), selecionando equações de volume para a Floresta Nacional do Tapajós, identificaram que o modelo de Spurr (Log) estava entre os dois que melhor se ajustaram aos dados.

A Figura 1 ilustra as distribuições dos volumes observados versus volumes estimados e dos resíduos em função do DAP para as duas equações selecionadas, com base no VP. A dispersão gráfica foi muito semelhante entre as equações e não mostrou tendências nítidas, o que indica que os critérios estatísticos adotados podem favorecer uma seleção adequada dos modelos para a estimativa do volume total. Todavia, o modelo 7 apresentou menor amplitude relativa dos resíduos (AR) $(28,02 \%)$ em relação ao modelo 1 (36,75\%), o que sugere que o modelo de Spurr ( $\mathrm{Log}$ ) (Modelo 7) seria mais adequado para estimar a variável de interesse. Mazzonetto et al. (2012) e Melo et al. (2013), testando o ajuste de equações volumétricas para $P$. taeda e para $P$. caribaea, respectivamente, observaram que o modelo de Spurr (Log) esteve entre os que melhor descreveram o volume das espécies analisadas, com pequena variação e menores tendências.

\section{Modelo 1}
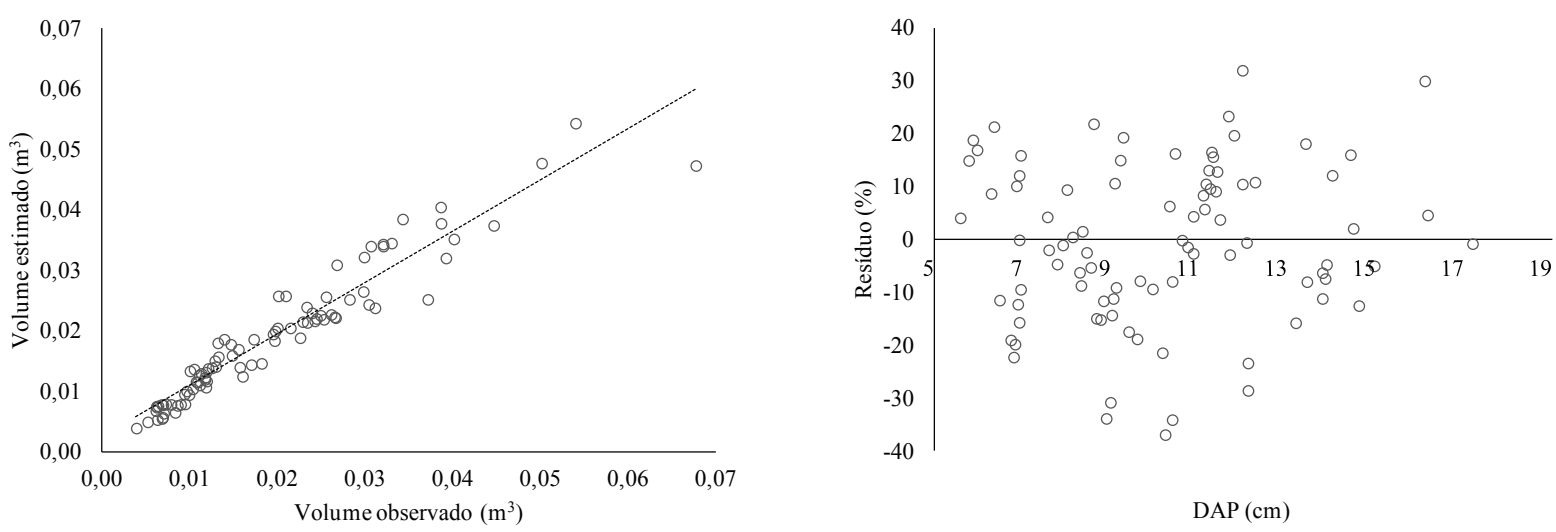

Modelo 7
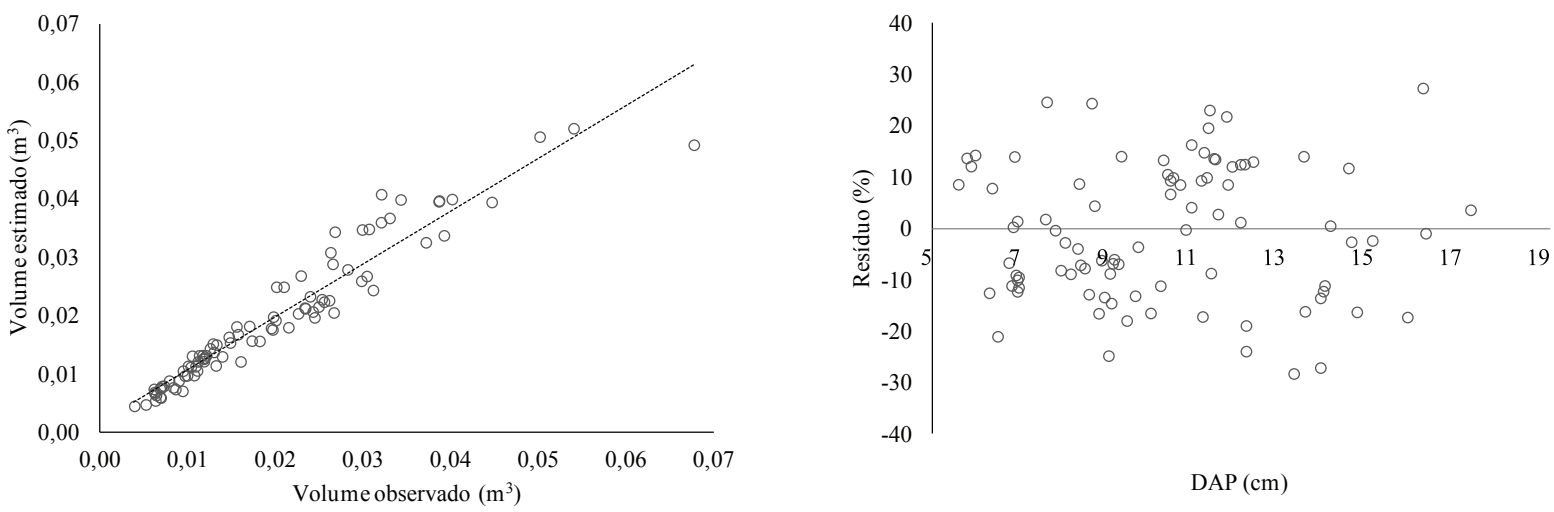

Figura 1. Distribuição dos volumes observados versus estimados e dos resíduos em função do diâmetro a 1,30 m do nível do solo (DAP) de Genipa americana para os modelos selecionados (Modelo 1: Hush e Modelo 7: Spurr (Log)). 
O fator de forma médio para o volume total foi 0,40 , o que indica que as árvores apresentam troncos com forma pouco cilíndrica. Tonini et al. (2005), estudando a volumetria de espécies nativas, encontraram fator de forma médio de 0,38 para jatobá (Hymenaea courbaril) e de 0,46 para Ipê roxo (Tabebuia avellanedae). Resultado semelhante foi observado por Azevedo et al. (2011), que estudando métodos de estimativa de volume de Eucalyptus urophylla com cinco anos, na mesma região de estudo do presente trabalho, obtiveram valor médio de fator de forma de 0,49 .

Quando analisadas pelo teste t pareado, as diferenças entre as estimativas obtidas pelo fator de forma e pelos dois modelos em relação aos respectivos valores reais foram consideradas não significativas (Tabela 4), apresentando erro padrão da diferença média entre 0,0009 (Modelo 7) e 0,0027 (Fator de forma). Ou seja, os dois métodos propiciaram estimativas estatisticamente semelhantes aos seus valores reais. Todavia, observa-se que, em média, o erro associado às estimativas obtidas pelas equações (modelo $1=2,01 \%$ e modelo $7=$ $0,40 \%$ ) foi menor que o erro obtido pelo fator de forma $(22,68 \%)$ (Tabela 4). Dessa forma, pode-se afirmar que as equações ajustadas apresentam superioridade sobre o fator de forma.

Tabela 4. Comparação de médias de volume individual de árvores de Genipa americana obtidas pela cubagem e estimadas pelo fator de forma e modelos ajustados.

\begin{tabular}{ccccc}
\hline Volume & Média & $\mathbf{p}$ & $\mathbf{E P}$ & $\mathbf{E}(\%)$ \\
\hline Cubagem & 0,01781 & - & - & - \\
Modelo 1 & 0,01737 & $0,67377^{\text {ns }}$ & 0,00101 & 2,01 \\
Modelo 7 & 0,01774 & $0,93723^{\text {ns }}$ & 0,00088 & 0,40 \\
Fator de forma & 0,02185 & $0,16982^{\text {ns }}$ & 0,00271 & 22,68 \\
\hline
\end{tabular}

Em que: $\mathrm{p}=$ nível crítico observado pelo teste $\mathrm{t}$ de Student em relação a cubagem; ns = não significativo ao nível de 1\% de significância; $\mathrm{EP}=$ erro padrão da diferença média; $\mathrm{E}(\%)=$ erro médio associado à estimativa em percentual; Modelo $1=$ Hush; Modelo $7=\operatorname{Spurr}(\log )$.

De acordo com Miguel et al. (2010) e Soares et al. (2011), o fator de forma é considerado uma ferramenta de grande valia para obtenção de estimativas rápidas da variável volume, porém a sua precisão costuma ser inferior às equações de volume, o que requer atenção quanto ao seu uso. Fraga et al. (2014), comparando três métodos para obtenção de estimativas de volume de árvores de Pterogyne nitens, também observaram maior desempenho das equações ajustadas em relação ao fator de forma.
Apesar do modelo 7 ter propiciado menor erro associado à estimativa, cabe salientar que, como a determinação da altura das árvores é uma operação onerosa e sujeita a erros, a utilização do modelo 1 (Hush) pode representar redução de tempo e custos das operações de inventário florestal, uma vez que esse modelo adota apenas o DAP como variável independente, sendo, portanto, mais recomendado para a obtenção de estimativas de volume total da espécie nas condições estudadas.

\section{Conclusões}

Dentre os métodos utilizados para estimar o volume, o mais recomendado é o emprego de equações volumétricas ajustadas.

O modelo de Spurr (Log) é o mais indicado para estimar o volume total de árvores com casca de Genipa americana nas condições estudadas. Entretanto, o modelo de Hush também proporcionou estimativas próximas aos valores reais e, dessa forma, a sua utilização pode representar redução de tempo e custos das atividades em campo, uma vez que esse modelo estima o volume apenas em função do DAP.

\section{Referências}

AZEVEDO, G. B.; SOUSA, G. T. O.; BARRETO, P. A. B.; CONCEIÇÃO JÚNIOR, V. Estimativas volumétricas em povoamentos de eucalipto sob regime de alto fuste e talhadia no sudoeste da Bahia. Pesquisa Florestal Brasileira, Colombo, v. 31, n. 68, p. 309-318, out./dez. 2011. DOI: 10.4336/2011.pfb.31.68.309.

BARROS, D. A.; MACHADO, S. A.; ACERBI JUNIOR, F. W.; SCOLFORO, J. R. S. Comportamento de modelos hipsométricos tradicionais e genéricos para plantações de Pinus oocarpa em diferentes tratamentos. Boletim Pesquisa Florestal, Colombo, n. 45, p. 3-28, 2002.

CARVALHO, S. P. C. C.; CALEGARIO, N.; SILVA, F. F.; BORGES, L. A. C.; MENDONÇA, A. R.; LIMA, M. P. Modelos não lineares generalizados aplicados na predição da área basal e volume de Eucaliptus clonal. Cerne, Lavras, v. 17, n. 4, p. 541-548, 2011. DOI: 10.1590/S0104-77602011000400013.

FINGER, C. A. G. Fundamentos de biometria florestal. Santa Maria, RS: UFSM/CEPEF/FATEC, 1992. 269 p.

FRAGA, M. P.; BARRETO, P. A. B.; PAULA, A. Estimação de volume de Pterogyne nitens em plantio puro no sudoeste da Bahia. Pesquisa Florestal Brasileira, Colombo, v. 34, n. 79, p. 207-215, 2014. DOI: 10.4336/2014.pfb.34.79.703.

GUIMARÃES, D. P.; LEITE, H. G. Influência do número de árvores na determinação de equação volumétrica para Eucalyptus grandis. Scientia Forestalis, Piracicaba, n. 50, p. 37-42, dez. 1996. 
HIGUCHI, N.; SANTOS, J. dos; RIBEIRO, R. J.; MINETTE, L.; BIOT, Y. Biomassa da parte aérea da vegetação de floresta tropical úmida de terra-firme da Amazônia Brasileira. Acta Amazônica, Manaus, v. 28, v. 2, p. 153-165, 1998.

KOHLER, S. V.; KOEHLER, H. S.; FIGUEIREDO FILHO, A. Modelos de afilamento para Pinus taeda por classes de idade. Floresta e Ambiente, Seropédica, v. 20, n. 4, p. 470-479, 2013. DOI: 10.4322/floram.2013.039.

LORENZI, H. Árvores brasileiras: manual de identificação e cultivo de plantas arbóreas nativas do Brasil. 4. ed. Nova Odessa: Instituto Plantarum, 2002. $367 \mathrm{p}$.

MACHADO, S. A.; CONCEIÇÃO, M. B.; FIGUEIREDO FILHO, A. Modelagem do volume individual para diferentes idades e regimes de desbaste em plantações de Pinus oocarpa. Guarapuava. Revista Ciências Exatas e Naturais, v. 4, n. 2, p. 41- 50, 2002.

MACHADO, S. A.; MELLO, J. M.; BARROS, D. A. Comparação entre métodos para avaliação de volume total de madeira por unidade de área, para o pinheiro do Paraná, na região sul do Brasil. Cerne, Lavras, v. 6, n. 2, p. 55-66, 2000.

MARTINS, R. M.; LEITE, M. V. S.; CABACINHA, C. D.; ASSIS, A. L. Teste de identidade de modelos volumétricos para povoamentos de Eucalyptus sp. Em sete municípios de Minas Gerais. Enciclopédia Biosfera, Goiânia, v. 11, n. 21, 2015.

MAZZONETTO, C. R.; FRACASSO, R. M.; FORTES, F. Avaliação de modelos na estimativa de volume de Pinus taeda. In: CONGRESSO FLORESTAL ESTADUAL DO RS; SEMINÁRIO MERCOSUL DA CADEIA MADEIRA, 2., Nova Prata, Rio Grande do Sul. Anais... Nova Prata: UFSM/DCFL, 2012. p. 562-567.

MELO, L. C.; BARRETO, P. A. B.; OLIVEIRA, F. G. R. B.; NOVAES, A. B. Estimativas volumétricas em povoamentos de Pinus caribaea var. hondurensis no Sudoeste da Bahia. Pesquisa Florestal Brasileira, Colombo, v. 33, n. 76, p. 379-386, 2013. DOI: 10.4336/2013.pfb.33.76.459.

MIGUEL, E. P.; CANZI, L. F.; RUFINO, R. F.; SANTOS, G. A. Ajuste de modelo volumétrico e desenvolvimento de fator de forma para plantios de Eucalyptus grandis localizados no município de Rio Verde - GO. Enciclopédia Biosfera, Goiânia, v. 6, n. 11, 2010.

MIGUEL, E. P.; LEAL, F. A.; ONO, H. A.; LEAL, U. A. S. Modelagem na predição do volume individual em plantio de Eucalyptus urograndis. Revista Brasileira de Biometria, São Paulo, v. 32, n. 4, p. 584-598, 2014.

OLIVEIRA, C. V.; BARRETO, P. A. B.; GOMES, A. S.; GUIMARÃES, S. O. Efeito de borda e decomposição da serapilheira foliar de um fragmento florestal, em Vitória da Conquista - BA. Enciclopédia Biosfera, Goiânia, v. 9, n. 17, 2013.
OLIVEIRA, L. M.; SILVA, E. O.; BRUNO, R. L. A.; ALVES, E. U. Períodos e ambientes de secagem na qualidade de sementes de Genipa americana L. Semina: Revista Ciências Agrárias, Londrina, v. 32, n. 2, p. 495-502, 2011. DOI: 10.5433/1679-0359.2011v32n $2 \mathrm{p} 495$.

RANGEL, M. S.; CALEGARIO, N.; MELLO, A. A., LEMOS, P. C. Melhoria na precisão da prescrição de manejo para floresta natural. Cerne, Lavras, v. 12, n. 2, p. 145-156, 2006.

REZENDE, A. V.; VALE, A. T.; SANQUETTA, C. R.; FIGUEIREDO FILHO, A.; FELFILI, J. M. Comparação de modelos matemáticos para estimativa do volume, biomassa e estoque de carbono da vegetação lenhosa de um cerrado sensu stricto em Brasília, DF. Scientia Forestalis, n. 71, p. 65-76, 2006.

RIBEIRO, R. B. S.; GAMA, J. R. V.; MELO, L. O. Seccionamento para cubagem e escolha de equações de volume para a Floresta Nacional do Tapajós. Cerne, Lavras, v. 20, n. 4, p. 605-612, 2014. DOI: 10.1590/01047760201420041400.

ROCHA, T. B.; CABACINHA, C. D.; ALMEIDA, R. C.; DE PAULA, A. SANTOS, R. C. Avaliação de métodos de estimativa de volume para um povoamento de Eucalyptus urophylla S. T. Blake no Planalto da Conquista - BA. Enciclopédia Biosfera, Goiânia, v. 6, n. 10, 2010.

SCOLFORO, J. R. S.; FIGUEIREDO FILHO, A. Biometria florestal: medição e volumetria de árvores. Lavras: UFLA/FAEPE, $1998.310 \mathrm{p}$.

SCOLFORO, J. R. S.; PÉREZ, J. F. M.; MELLO, J. M. de; OLIVEIRA, A. D. de; CAMOLESI, J. F.; BORGES, L. F. R.; ACERBI JÚNIOR, F. W. Estimativas de volume, peso seco, peso de óleo e quantidade de moirões para a Candeia (Eremanthus erythropappus (DC.) MacLeish). Cerne, Lavras, v. 10, p. 87-102, 2004.

SOARES, C. P. B.; PAULA NETO, F.; SOUZA, A. L. Dendrometria e inventário florestal. 2. ed. Viçosa, MG: Ed. da UFV, 2011. 270 p.

THIERSCH, A. A eficiência das distribuições diamétricas para prognose de Eucalyptus camaldulesis. 1997. 155 f. Dissertação (Mestrado em Engenharia Florestal) - Universidade Federal de Lavras, Lavras.

TONINI, H.; ARCO-VERDE, M. F.; SÁ, S. P. P. Dendrometria de espécies nativas em plantios homogêneos no Estado de Roraima Andiroba (Carapa guianensis Aubl), Castanha-do-Brasil (Bertholletia excelsa Bonpl.), Ipê-roxo (Tabebuia avellanedae Lorentz ex Griseb) e Jatobá (Hymenaea courbaril L.). Acta Amazonica, Manaus, v. 35, n. 3, p. 353-362, 2005. DOI: 10.1590/S0044-59672005000300008. 
